\title{
COVID-19: Special Precautions in Dentistry
}

\author{
Adel Bouguezzi ${ }^{1,2 *}$, Imen Cherni ${ }^{1,2}$, Sameh Sioud ${ }^{1,2}$, Hajer Hentati ${ }^{1,2}$ and Jamil Selmi ${ }^{1,2}$ \\ ${ }^{1}$ Faculty of Dental Medicine, Oral Health and Oro-Facial Rehabilitation Laboratory Research (LR12ES11), University of \\ Monastir, Tunisia
}

${ }^{2}$ Dental Clinic of Monastir, Department of Medicine and Oral Surgery, Tunisia

\begin{abstract}
A novel coronavirus (COVID-19) is associated with human-to-human transmission. Due to the unique nature of dentistry, most dental procedures generate significant amounts of droplets and aerosols, posing potential risks of infection transmission, the routes of transmission are direct contact, and droplet and possible aerosol transmissions. The aim of this article is to provide specific recommendations for dental practice and patient management protocol.
\end{abstract}

KEYWORDS: Corona virus disease 2019; Infection control; Dental practice management

\section{SHORT COMMUNICATION}

\section{Why Dentistry is a Risk Branch?}

Dental patients and professionals can be exposed to pathogenic microorganisms, including viruses and bacteria that infect the oral cavity and respiratory tract. Dental care settings invariably carry the risk of 2019-nCoV infection due to the specificity of its procedures, which involves face-to-face communication with patients, and frequent exposure to saliva, blood, and other body fluids, and the handling of sharp instruments. The pathogenic microorganisms can be transmitted in dental settings through inhalation of airborne microorganisms that can remain suspended in the air for long periods [1], direct contact with blood, oral fluids [2], or other patient materials, contact of conjunctival, nasal, or oral mucosa with droplets and aerosols containing microorganisms generated from an infected individual and propelled a short distance by coughing and talking without a mask [3], and indirect contact with contaminated instruments and/or environmental surfaces [1]. Infections could be present through any of these conditions involved in an infected individual in dental clinics and hospitals, especially during the outbreak of 2019-nCoV.

\section{Strategies to Reduce Droplet Generation in Different Dental Disciplines}

Up to now, there has been no consensus on the provision of dental services during the epidemic of COVID-19. Based on relevant guidelines and research (Guideline for the Diagnosis and Treatment of Novel Coronavirus Pneumonia (the $5^{\text {th }}$ edition) [4] and the Guideline for the Use of Medical Protective Equipment in the Prevention and Control of Novel Coronavirus Pneumonia [5] released by the National Health Commission of the People's Republic of China, certain specific measures are discussed here for dental patient management in this epidemic period of COVID-19.

Preprocedural mouth rinse: Although the effect of preprocedural mouth rinse against coronavirus is still unknown, it has been proven that CHX is effective against several infectious viruses, including herpes simplex virus (HSV), human immunodeficiency virus (HIV), and hepatitis B virus (HBV) [6].

Endodontics: Rubber dam must be applied during endodontic treatment Root canal treatment usually requires several endodontic
Quick Response Code:

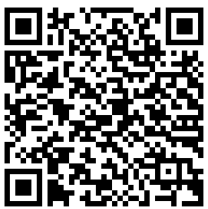

Address for correspondence: Adel Bouguezzi, University of Monastir, Faculty of Dental Medicine, Oral Health and Oro-Facial Rehabilitation Laboratory Research (LR12ES11), Tunisia

Received: April 15, 2020 Published: April 21, 2020

How to cite this article: Adel B, Imen C, Sameh S, Hajer H, Jamil S. COVID-19: Special Precautions in Dentistry. 2020 - 2(2). OAJBS.ID.000164. DOI: 10.38125/OAJBS.000164 
instruments and devices, therefore minimizing unnecessary hand contact with surfaces and equipment in the dental office to reduce possibility of fomite transmission [7].

Restorative dentistry and pediatric dentistry: Avoid using rotary instruments during cavity preparation. In selective cases, consider using chemochemical caries removal or atraumatic restorative techniques If rotary instrumentation must be performed, rubber dam isolation should be applied.

Periodontics: Hand and ultrasonic instrumentation are equally effective in removing plaque and calculus deposits; if required, manual scaling and polishing are recommended [8].

Prosthodontics: Salivary suction must be performed with care to avoid gagging Select and adjust trays to the right size for impression taking to avoid cough reflex. For highly sensitive patients, consider applying oral mucosa anesthesia to the throat before impression taking During fixed partial denture or singlecrown preparation, treatment alternation may be considered to incorporate rubber dam application. For example, design supragingival margin for posterior bridge or use a split-dam technique [7]. During removable partial denture or complete denture try-in, avoid touching other objects in the dental office after contacting patients' saliva Upon removal from patient's mouth, dental prosthesis, impressions, and other prosthodontics materials (e.g., bite registration) should be thoroughly disinfected by a disinfectant having at least intermediate level activity.

Oral-maxillofacial surgery: When performing simple extraction, treat the patient in a supine position to avoid working in the breath way of a patient [7].

\section{CONCLUSION}

Dentists, by nature, are at high risk of exposure to infectious diseases. The emergence of COVID-19 has brought new challenges and responsibilities to dental professionals. A better understanding of aerosol transmission and its implication in dentistry can help us identify and rectify negligence in daily dental practice. In addition to the standard precautions, implementation of special precautions could prevent disease transmission from asymptomatic carriers. These special precautions would not only help control the spread of COVID-19 but also serve as a guide for managing other respiratory diseases.

\section{REFERENCES}

1. Liu L (2011) Epithelial cells lining salivary gland ducts are early target cells of severe acute respiratory syndrome coronavirus infection in the upper respiratory tracts of rhesus macaques. J Virol 85(8): 4025-4030.

2. To KK (2020) Consistent detection of 2019 novel coronavirus in saliva. Clin Infect Dis.

3. Chen J (2020) Pathogenicity and transmissibility of 2019-nCoV-a quick overview and comparison with other emerging viruses. Microb Infect 22(2): 69-71.

4. The guideline for the diagnosis and treatment of novel-coronavirus pneumonia (the $5^{\text {th }}$ edition).

5. The guideline for the use of medical protective equipment in the prevention and control of novel coronavirus pneumonia.

6. Souid K, Bouguezzi A, Hasni W, Zaghbani A, Ben Youssef S, et al. (2013) Intérêt de la chlorhexidine à $0,12 \%$ et l'hypochlorite de sodium à 0,5 $\%$ en phase pré-opératoire: Etude comparative. Médecine Buccale Chirurgie Buccale 19(4): 221-226.

7. Peng X, Xu X, Li Y (2020) Transmission routes of 2019-nCoV and controls in dental practice. Int J Oral Sci 12(1): 9.

8. Krishna R, De Stefano JA (2016) Ultrasonic vs. hand instrumentation in periodontal therapy: clinical outcomes. Periodontology 71(1): 113-127. 